\title{
Discovering hair biomarkers of Alzheimer's disease using high resolution mass spectrometry-based untargeted metabolomics
}

Pao-Chi Liao ( $\square$ liaopc@mail.ncku.edu.tw)

National Cheng Kung University

Chih-Wei Chang

National Cheng Kung University

Shih-Wen Li

National Cheng Kung University

Pi-Shan Sung

National Cheng Kung University Hospital, National Cheng Kung University

Ru-Hsueh Wang

National Cheng Kung University Hospital, National Cheng Kung University

Chih-Hsing Wu

National Cheng Kung University Hospital, National Cheng Kung University

Yuan-Chi Chen

National Cheng Kung University

\section{Research Article}

Keywords: Alzheimer's disease, untargeted metabolomics, hair, biomarker discovery, high-resolution mass spectrometry

Posted Date: February 25th, 2022

DOI: https://doi.org/10.21203/rs.3.rs-1388089/v1

License: (a) (1) This work is licensed under a Creative Commons Attribution 4.0 International License. Read Full License 


\section{Abstract}

Background: Alzheimer's disease (AD) is a common and progressive neurodegenerative disorder related to cognitive impairments that generally include deficits in short-term memory. Hair may be a potential biospecimen for $A D$ biomarker discovery since it reflects the integral metabolic profiles of body burden over several months. Here, we discovered hair biomarkers in hair that differentiate AD patients from cognitively healthy controls using a high-resolution mass spectrometry (HRMS)-based untargeted metabolomics approach.

Methods: An HRMS-based untargeted metabolomics approach was used to discover the potential AD biomarker candidates in hair samples from 24 AD patients (including $n=14,7$, and 3 , with CDR scores of $0.5,1$, and 2 , respectively) and 24 age- and sex-matched cognitively healthy controls. The hair samples were collected $0.1-\mathrm{cm}$ away from the scalp and further cut into $3-\mathrm{cm}$ segments. Hair metabolites were extracted by ultrasonication with methanol/phosphate-buffered saline 50/50 (v/v) for 4 hours. We assessed metabolic biomarker to distinguish between $A D$ and controls with Student's t test, hierarchical cluster analysis (HCA), and receiver operating characteristic (ROC) curve analysis.

Results: Statistically significant changes in 61 peak features in hair between the patients with AD and cognitively healthy controls were discovered, of which 25 were identified to have chemical structures. The levels of nine metabolites, including acetyl-L-carnitine, propionylcarnitine, butyrylcarnitine, O-valeroyl-Lcarnitine, PC (16:0/0:0), LPC 18:1, piperine, hydroxyprolyl-leucine, and 6-0-methylnorlaudanosoline, were significantly lower in patients with questionable AD (CDR score of 0.5 ) than in cognitively healthy controls $(p<0.01)$. ROC curves were constructed to evaluate the performance of the nine metabolites combined into a metabolic marker panel for distinguishing patients with questionable AD from cognitively healthy controls, in which the sensitivity and specificity were $86 \%$ and $71 \%$, respectively, and the AUC value was 0.85 (95\% Cl: 0.72 0.97), indicating high potential for the initiation of AD dementia.

Conclusions: Nine metabolites may be used as biomarkers for the early detection of AD dementia or therapeutic development after validation in a larger cohort. The present study suggested that the hair metabolome can be used to reveal metabolic perturbations for biomarker discovery and may lead to the development of a sensitive diagnostic tool for AD.

\section{Background}

Alzheimer's disease (AD) is a progressive neurodegenerative disorder related to cognitive impairments that generally include deficits in short-term memory and executive and visuospatial dysfunctions and is thought to account for $60-80 \%$ of dementia cases [1]. Physicians can diagnose $A D$ through gradual onset of cognitive and behavioral symptoms, medical history, and a cognitive function test [2]. As cognitive deficits and behavioral abnormalities have been difficult to manifest in the early stage of $A D$, conducting early diagnosis and $A D$ onset prediction, biomarkers are needed to obtain additional insights into the etiology of $A D$ symptoms. In addition, $A D$ dementia is difficult to distinguish from other dementia- 
causing pathologies since some AD symptoms are similar to those of other diseases. Progressively accumulating extracellular amyloid-beta $(A \beta)$ plaques and intracellular neurofibrillary tangles formed by hyperphosphorylated tau protein are specific key proteins and considered two pathological hallmarks reflecting $A D$ pathology [3]. Quantifying biomarker levels in cerebrospinal fluid (CSF) and measuring $A \beta$ levels using positron-emission tomography imaging have been used to diagnose $A D$; however, it is very difficult to obtain CSF samples, and these processes are expensive for employment in the clinical practice [4-6].

Untargeted metabolomics in hair may be a novel approach for discovering novel diagnostic markers and developing new potential therapeutic targets in AD. Metabolomics is the study of the small-molecule composition within biological systems, and it has been applied to multiple fields [7]. The human metabolome is estimated to contain at least 220,000 compounds, and a large fraction of metabolites remain unidentified [8]. CSF and blood are the most conventional biological specimens used to identify $\operatorname{AD}$ metabolic biomarkers $[3,9,10]$. The procedures requiring collection of CSF samples are too invasive for routine clinical use. As urine and blood metabolomes are affected by circadian rhythms, daily activities, and sleep patterns, the concentrations of chemical compounds fluctuate daily [11-13]. Recently, the use of hair has been shown to be important for the long-term accumulation of both environmental substance exposure and endogenous metabolite perturbations and has been applied to disease biomarker discovery [14-16]. Hair segments of $1 \mathrm{~cm}$ reflect the metabolic profile over the past month, as they grow $1 \mathrm{~cm}$ per month $[17,18]$. Chemical compounds in blood capillaries connected to hair roots are incorporated into the hair via passive diffusion [19-21]. The longitudinal distribution of chemical compound concentrations along the hair shaft reflects changes in the corresponding composition during the measurement period [20].

The human hair metabolome might be used for discovering novel biomarkers of AD since it can reflect the metabolic profiles of body burden over several months.

As distinguishing AD from other causes of dementia based on apparent symptoms is often difficult, discovering hair biomarkers may be capable of diagnosing AD during the early stage and monitoring its progression is an important strategy for managing the disease. Here, we performed an ultrahighperformance liquid chromatography-high-resolution mass spectrometry (UHPLC-HRMS)-based untargeted metabolomic approach on hair samples from AD patients and subjects with normal cognition serving as controls. We investigated the perturbations in the hair metabolome between AD patients and cognitively healthy controls and further discovered potential metabolic biomarkers to distinguish AD patients from cognitively healthy controls.

\section{Methods}

\section{Study design}


The study design for discovering hair AD biomarkers is depicted in Figure 1. The participants were recruited from National Cheng Kung University Hospital, Tainan, Taiwan. Hair samples were collected 0.1$\mathrm{cm}$ away from the scalp from 24 AD patients and 24 age- and sex-matched cognitively healthy controls. Hair metabolites were extracted by an established and optimized procedure described in a previous study [22]. The hair extracts were subjected to UHPLC-HRMS analysis with full scan and the raw data were processed with Progenesis QI software, which yielded a peak detection and alignment table. Fold change and Student's $t$ test were performed to identify differential levels of peak features. We used the online available databases of Massbank of North America (MoNA) with MS-DIAL and MS-Finder software to identify the chemical structures of potential biomarkers.

\section{Participant description}

The 48 participants included adults 40 years or older without a history of stroke or known malignancy. AD patients $(n=24)$ recruited from the Department of Neurology in National Cheng Kung University Hospital, Tainan, Taiwan were diagnosed based on professional physicians. The Montreal Cognitive Assessment (MoCA) was performed, and the severity of the symptoms of dementia was assessed by neurologists using the clinical dementia rating (CDR). Age- and sex-matched control subjects $(n=24)$ recruited from the Department of Family Medicine in National Cheng Kung University Hospital, Tainan, Taiwan underwent MoCA and had a MoCA score $\geq 26$ points. Other important information on age, body mass index (BMI), cosmetic hair treatment, smoking status, and alcohol consumption was obtained and is shown in Table 1. This study was approved by the Ethics Committee of National Cheng Kung University Hospital. All participants in the study signed a written informed consent under the rules and requirements of the Institutional Review Board of National Cheng Kung University Hospital (IRB approval no. B-ER-108188).

\section{Hair sampling and metabolite extraction}

Hairs were cut $0.5-\mathrm{cm}$ away from the scalp with scissors, secured in aluminum foil and stored at $4{ }^{\circ} \mathrm{C}$ until further analysis. The washing procedure was performed according to the Society of Hair Testing guidelines [23] as a mandatory step to remove contaminants deposited on the hair shaft. A six-milligram $3-\mathrm{cm}$ hair sample was weighed in a glass tube and washed with $1.8-\mathrm{mL}$ acetone followed by deionized 1.8- $\mathrm{mL}$ water in an ultrasonic bath for $2 \mathrm{~min}$ each. The washing solutions were then discarded, and the samples were dried under nitrogen for $90 \mathrm{~min}$. Afterward, the dried hair samples were cut into round 0.2$\mathrm{cm}$ snippets with a pair of scissors and subjected to the extraction procedure. The samples were mixed with 300- $\mu \mathrm{L}$ methanol $(\mathrm{MeOH}) /$ phosphate-buffered saline (PBS) $(50 / 50, \mathrm{v} / \mathrm{v})$ and underwent sonication for 4 hours under $55^{\circ} \mathrm{C}$. The extracts were centrifuged at $15000 \times \mathrm{g}$ for $15 \mathrm{~min}$ and the supernatants were collected. The hair extract was subsequently evaporated to dryness by speed vac and the residue was

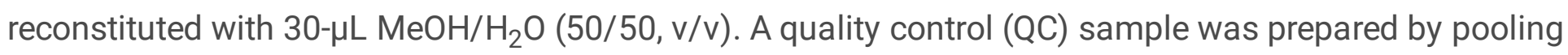
$5 \mathrm{mg}$ of each hair sample from 48 participants and subjected to the extraction procedure. QC samples 
were prepared in parallel with study samples and analyzed after 4 sample injections to monitor the robustness of the large-scale analysis.

\section{UHPLC-HRMS analysis}

A UHPLC system coupled with an Orbitrap HRMS system (Thermo Fisher Scientific) was used for the hair sample analysis. The chromatographic separation was performed by a Waters Acquity UPLC BEH C18 column $(2.1 \mathrm{~mm} \times 100 \mathrm{~mm}, 1.7 \mu \mathrm{m})$. The mobile phases were composed of (A) $2 \%$ ACN in deionized water with $0.1 \%$ formic acid and (B) acetonitrile with $0.1 \%$ formic acid. The gradient conditions were as follows: 0-1 min, 2\% B; 1-11 min, 2\%-99\% B; 11-13 min, 99\% B; 13-13.01 min, 99\%-2\% B; 13.01-14 min, $2 \% \mathrm{~B}$. The flow rate was set at $250 \mu \mathrm{L} / \mathrm{min}$. The column temperature was kept at $40{ }^{\circ} \mathrm{C}$ and the injection volume was $5 \mu \mathrm{L}$.

The Q Exactive Orbitrap and Lumos Orbitrap were operated in positive and negative modes, respectively. The mass range was $\mathrm{m} / \mathrm{z} 100$ to 1000 and the resolution was 70,000 and 60,000 in MS1 using a Q Exactive Orbitrap and Lumos Orbitrap, respectively. The MS/MS operating in a targeted mass list was adopted for fragmentation by higher-energy collisional dissociation (HCD) with a normalization collision energy of $50 \%$.

\section{Data processing, statistical analysis, and metabolite identification}

The raw data were analyzed by Progenesis QI software, performing peak detection and alignment. Peak tables containing accurate masses, retention times, and peak abundances were exported. The features of $\mathrm{S} / \mathrm{N}<3$ were considered as the absence of peaks and were hence filtered. Before performing the univariate analysis to discover the differential features, each raw abundance was normalized by dividing by the sum of the raw abundances of all peaks in the corresponding sample. Volcano plot and hierarchical cluster analyses were conducted by $\mathrm{R}$ and Metaboanalyst, respectively, [24] to visualize the discriminatory features and the clustering pattern of the hair samples and metabolites, respectively. Discriminatory features were selected for tandem mass spectrometry (MS/MS) analysis using a Thermo Fusion Lumos Orbitrap mass spectrometer (Thermo Fisher Scientific, San Diego, CA) operated in both positive and negative ion mode with the same chromatographic conditions described in the previous section. We performed mass spectral similarity searches of the experimental to MoNA database within MS-DIAL and generated fragments calculated on candidates retrieved from available compound databases within MS-Finder [25]. Search windows were performed within $5 \mathrm{ppm}$ mass tolerance for the precursor masses. Receiver operating characteristic (ROC) curves were plotted to evaluate the predictive performance of the model, and random forest analysis was performed to generate a metabolite panel.

\section{Results}




\section{Characteristics of the case and control groups}

Table 1 presents the basic demographic characteristics of 48 participants recruited from the National Cheng Kung University Hospital, Tainan, Taiwan. The case group included 24 patients with AD, and the control group consisted of 24 age- and sex-matched subjects without AD (MoCA score $\geq 26$ ).

Participants with a stroke history or known malignancy were excluded from this study. AD patients were diagnosed based on DSM-5 criteria by professional physicians from the Department of Neurology. The mean MoCA scores for AD patients and control subjects were $15.5(S D=5.3)$ and $27.4(S D=1.4)$, respectively. The severity of the dementia symptoms was assessed by neurologists using the CDR scale, which showed fourteen patients with questionable $A D$ (CDR score of 0.5), seven patients with mild $A D$ (CDR score of 1), and three had moderate AD (CDR score of 2) [26]. A comparison between the demographic variables of the AD patients and controls showed no statistically significant difference in age (mean $=68.7$ and 66.2 years, respectively, $p=0.17$ ), $\mathrm{BMI}$ (mean $=24.2$ and $23.9 \mathrm{~kg} / \mathrm{m}^{2}$, respectively, $p$ $=0.83)$, cosmetic hair treatment $(p=0.93)$, smoking status $(p=0.33)$, or alcohol consumption $(p=0.60)$.

\section{Discovery and identification of AD biomarkers}

All 3-cm hair segments were subjected to the sample preparation procedure and subsequently analyzed by UHPLC-HRMS in positive and negative ion modes, resulting in 12,358 and 8,821 aligned peak features extracted from the corresponding data. The relative standard deviation (RSD) of the peak area of all detected signals within the QC sample prepared by pooling aliquots from all the individual samples is shown in Figure S1A, resulting in a calculated median RSD of $46 \%$ while $60 \%$ of the total detected peaks had RSD values below $56 \%$ during the measurement.

Two volcano plots shown in Figure $2 \mathrm{~A}$ and $\mathrm{B}$ were generated to reveal significant signal intensity changes ( $\mid$ log2 (fold-change) $\mid \geq 1$ and $p$ value $\leq 0.01$ ) of peak features between the $A D$ and control groups in positive and negative UHPLC-HRMS ion modes, respectively, suggesting 72 discriminatory features (Table S1). Among these, 32 peak features indicated overexpression and 40 indicated low expression in AD patients relative to the levels in the control subjects. The RSD of the peak area of the 72 discriminatory peak features within QC samples and its replicate for 12 samples are shown in Figure S1B, indicating that the 61 discriminatory peak features had RSD values below $50 \%$, suggesting that they were subjected to tandem mass spectrometry (MS/MS) analysis for chemical structure identification.

The 61 discriminatory peak features were subjected to MS/MS analysis for chemical structure identification, as shown in Table S2. We used MS-DIAL software to perform mass spectral similarity searches of experimental MS/MS results in the MoNA database. The chemical structures of ten biomarkers were identified through the MoNA database with MS-DIAL. In addition, we matched experimental MS/MS spectra against a variety of MS-FINDER-generated fragments calculated on candidate compounds retrieved from available databases, resulting in a total of 21 biomarkers was identified through MS/MS spectra generated by MS-FINDER. 
To find the discriminatory peak features with similar expression patterns in the 48 hair samples, unsupervised clustering of the detected normalized intensity of 25 annotated metabolites was performed by two-way hierarchical cluster analysis (HCA) based on Pearson's correlation, as shown in Figure 3. The 25 annotated metabolites could be grouped into 2 clusters (Clusters 1 and 2). Cluster 1 metabolites showed increased levels in AD patients compared to controls, and cluster 2 metabolites, including acetylL-carnitine, propionylcarnitine, butyrylcarnitine, O-valeroyl-L-carnitine, PC (16:0/0:0), LPC 18:1, piperine, pyridoxal, 6-0-methylnorlaudanosoline, and hydroxyprolyl-leucine, showed lower levels in patients with $A D$ than that in healthy controls. The 48 hair samples could be grouped into 10 clusters (Clusters $A \sim J$ ) based on 25 discriminatory metabolites with similar peak feature profiles. Clusters $\mathrm{E}$ to $\mathrm{J}$ primarily consisted of the cognitively healthy controls. Clusters $C$ and $D$ primarily included the 7 patients with questionable $A D$ and 4 patients with mild $A D$, suggesting that the levels of these metabolites might be used to group patients with questionable $A D$.

\section{Application of biomarkers for AD diagnosis}

As the 25 metabolites might be used to differentiate questionable AD from healthy controls, we further analyzed the normalized peak area of the metabolites from patients with different severities of $A D$ and healthy control groups using Student's t test (Figure 4 A J). It was revealed that 9 metabolites have significant differential levels between very mild $A D$ and healthy controls $(p<0.01)$, indicating high potential for the initiation or promotion phase of $A D$ dementia in the early stage. The receiver operating characteristic (ROC) curves of these 9 biomarker candidates between 14 questionable AD patients (CDR score of 0.5 ) and controls were plotted to further evaluate their performance as well the as area under the curve (AUC). These results are shown in Table 2, and AUC values of the 9 metabolite biomarkers ranged from 0.73 to 0.86 . Among these compounds, $6-0$-methylnorlaudanosoline has reached $90 \%$ sensitivity and $80 \%$ specificity in mild AD. Acetyl-L-carnitine and butyrylcarnitine were found to indicate AD in patients with approximately $80 \%$ sensitivity and at least $80 \%$ specificity. Propionylcarnitine and 0valeroyl-L-carnitine had approximately $70 \%$ sensitivity and specificity. Using a composite panel of these 9 biomarker candidates, the AUC value achieved 0.85 (95\% $\mathrm{Cl}: 0.72 \sim 0.97)$ in patients with very mild $A D$ compared to healthy controls. The combined diagnostic sensitivity and specificity of the composite panel in AD patients were $86 \%$ and $71 \%$, respectively (Figure 5), suggesting that these 9 biomarker candidates may be used for the early detection of $A D$.

We then further investigated the associations between these biomarker candidates and MoCA scores, and these results are shown in Figure S2 [3, 9]. Correlation coefficient values were $0.45(p=0.0014), 0.44$ $(p=0.0021), 0.44(p=0.0016), 0.43(p=0.0019)$, and $0.40(p=0.0064)$ between MoCA score and acetyl-Lcarnitine, propionyl carnitine, butyrylcarnitine, 0-valeroyl-L-carnitine, and 6-0-methylnorlaudanosoline, respectively, suggesting a moderate correlation $(r>0.4)$ between MoCA and the five metabolites (Figure $\mathrm{S} 2 \mathrm{~A} \sim \mathrm{E})$ [27].

\section{Discussion}


Chemical substances secreted from tissues or organs in different responses to physiological needs or stresses to the bloodstream are incorporated into the hair matrix during its growth [20,28-31]. Hair is an analytical biospecimen for the long-term accumulation of both xenobiotic exposure and endogenous perturbations, which may reflect the integral metabolic profiles of body burden over several months. A hair is not a homogeneous matrix but consists of keratinized cells that form three concentric structures: cuticle, cortex, and medulla. As the cells of hair follicles die and fuse to form hair strands, the chemical compounds are retained and accumulate in this extremely stable structure. The 25 discriminatory metabolites were discovered in hair between AD patients and controls using an HRMS-based untargeted metabolomics approach with good reproducibility. Donepezil and galantamine which are treatments for $A D$ dementia showed increased levels in AD patients compared to controls. They enable central cholinergic activity by inhibiting the physiological breakdown of acetylcholine by the enzyme acetylcholinesterase in synaptic gaps [32]. In addition to the detection of donepezil, increased levels of 6desmethyldonepezil, a metabolite of donepezil, were detected in the AD patients compared to those in the controls in this study.

We discovered that the combination of five metabolites, 6-0-methylnorlaudanosoline, acetyl-L-carnitine, propionylcarnitine, butyrylcarnitine, and O-valeroyl-L-carnitine, in hair could contribute to distinguishing mild AD patients from controls (Fig. 5), suggesting that they might be early markers of AD. A panel of biomarkers is likely to compensate for deficient sensitivity and specificity of a single marker used to distinguish AD patients from controls [9, 33, 34]. For example, when glutamine is combined with valine, a higher AUC value is generated, leading to an increased predictive power superior to that of glutamine or valine alone [33]. In our data, the discriminating performance of combining four acyl-carnitines and 6-0methylnorlaudanosoline was superior to that of any acyl-carnitine used alone. The observations of acylcarnitine concentrations in hair related to $A D$ pathology are not yet fully understood, although alterations in their concentrations in plasma and CSF have previously been reported. Decreased levels of acylcarnitines in plasma and CSF have been observed in $A D$ patients $[9,35,36]$. Acyl-carnitine can disrupt fatty acid transport into mitochondria for $\beta$-oxidation and further perturbations of energy metabolism in the brain, which is consistent with our observation that acyl-carnitines showed a lower expression level in $A D$ patients than in healthy subjects. An association between $A D$ and dysregulation of lipid metabolism, particularly perturbations in brain membrane lipids, has been implicated in AD pathology. It is reported that the increased level of glycerophosphocholine and choline generated by hydrolysis of PC in the CSF of patients with $A D$ compared to healthy controls [37,38]. Previous studies have shown that the levels of PC (16:0/0:0) and LPC 18:1 are significantly lower in patients with $A D$ than in healthy subjects $[39,40]$. Moreover, it is suggested that alterations in the metabolism of choline-containing phospholipids in the brain are closely associated with membrane changes in $A D$, which is consistent with our findings that reductions in PC (16:0/0:0) and LPC 18:1 levels were observed in AD patients compared to controls [41]. Studies showed lower levels of piperine in blood of patients with AD than that of healthy controls [42]. Piperine, the primary alkaloid constituent of black pepper, has been reported to attenuates cognitive impairment in an experimental mouse model of sporadic Alzheimer's disease [43]. 
In addition to our observations of evident differences between $A D$ and controls concerning acylcarnitines, phosphatidylcholines, and piperine, we also observed the novel AD biomarker 6-0methylnorlaudanosoline. The proposed mechanism by which 6-0-methylnorlaudanosoline influences AD pathogenesis involves a reduction in oxidative stress in the brain. It has been reported that 6-0methylnorlaudanosoline, a derivative of norlaudanosoline, reacts through catechol-0-methyltransferase in the brain [44]. The methylation of norlaudanosoline can increase its hydrophobicity and may enter the hair matrix through blood via passive diffusion. Norlaudanosoline, a dopaminergic neurotoxin, increases intracellular levels of reactive oxygen species (ROS) $[45,46]$, contributing to oxidative stress-induced synaptic dysfunction in $A D$ pathogenesis. In the brains of $A D$ patients, oxidative stress signatures are observed at the very early stage of the disease $[47,48]$. The neurotoxicity of norlaudanosoline may result from its tendency to autoxidize and yield reactive quinoids and subsequent hydroxyl radicals by redox cycling, further leading to oxidative stress-related cell death and DNA damage [49]. Moreover, norlaudanosoline derived from dopamine through condensation with 3,4-dihydroxyphenylacetaldehyde (dopaldehyde) is considered a dopaminergic neurotoxin and has been implicated in the pathology of Parkinson's disease $[50,51]$. It has been reported that norlaudanosoline evaluated in the urine of patients with Parkinson's disease treated with L-3,4-dihydroxyphenylalanine (L-DOPA) reduces tyrosine hydroxylase activity during L-DOPA production [52, 53]. Mono-0-methylated norlaudanosoline reduces the in vitro production of hydroxyl radicals during autoxidation in previous studies [54, 55]. Enzyme-mediated methylation reactions in the brain play vital roles in cells exposed to neuroprotective and neurotoxic components. The reduction in 6-0-methylnorlaudanosoline levels in AD patients might indicate that norlaudanosoline primarily undergoes spontaneous reactions to yield reactive quinoids and hydroxyl radicals, enhancing oxidative stress-induced neuronal apoptosis and dopamine degeneration. Therefore, 6-0-methylnorlaudanosoline may contribute to the underlying AD pathogenesis and be used for early detection of $A D$ in hair.

Some limitations of our study should be considered. First, in some experiments, the severity of phenotypes was not measured, such as the measurement of tau neurofibrillary or $A \beta$ plaques. The $A D$ patients were diagnosed only based on cognitive examination and professional neurological physicians. That is, AD was diagnosed based on observational evaluation of behavioral symptoms and memory impairment, but early symptoms in $A D$ are similar to those of other neurological disorders. In general, abnormal $A \beta$ and tau levels are used to identify $A D$; however, the measurement of $A \beta$ and tau in the CSF or through the use of positron-emission tomography imaging involves a complex sampling procedure and is expensive. Second, certain discriminatory features could not be identified as known metabolites or chemical formulas, creating a significant bottleneck in untargeted metabolomics research. Using a mass spectral database, MoNA, and the spectrum prediction program MS-Finder, we identified the chemical structure of $40 \%$ discriminatory features; however, more than $60 \%$ of the unknown chemical compounds might be important biomarkers of diseases. Third, we recognize that not all polar metabolites were comprehensively identified, as only reverse-phase liquid chromatography (RPLC) was employed. Perturbations in amino acids, bioenergetic metabolism, and redox imbalance are commonly observed in AD $[3,9,56]$. However, hydrophilic metabolites in the relevant pathways may be difficult to analyze by 
RPLC. A combination of RPLC and hydrophilic interaction liquid chromatography (HILIC) can ensure the global coverage of metabolites in an untargeted metabolomics investigation designed for AD biomarker discovery.

\section{Conclusions}

Nine discriminatory metabolites highlighted here may be used as biomarkers for early detection of $A D$ dementia or therapeutic development after validation in a larger cohort. Our study provides evidence that the hair metabolome shows a similar trend with the pathology of $A D$ and has great potential to reflect the long-term perturbations of oxidative stress, dopaminergic neurotoxin, mitochondrial $\beta$-oxidation metabolism, and dysregulation of lipids in AD. Investigation into the specific roles of the identified metabolic changes in the pathogenesis of $A D$ will offer novel insight into metabolic alterations. Besides, if validated in an external cohort of patients, the identified biomarker panel offers a promising way to detect early stage of Alzheimer's disease in a noninvasive manner.

\section{Abbreviations}

AD: Alzheimer's disease; AB: amyloid-beta plaques; AUC: area under the curve; BBB: blood-brain barrier; CSF: cerebrospinal fluid; CDR: clinical dementia rating; HCA: hierarchical cluster analysis; HILIC: hydrophilic interaction liquid chromatography; L-DOPA: 3,4-dihydroxyphenylalanine; lysoPC: lysophosphatidylcholine; MoNA: Massbank of North America; MoCA: Montreal Cognitive Assessment; MS/MS: tandem mass spectrometry; PC: phosphatidylcholine; ROC curve: receiver operating characteristic curve; ROS: reactive oxygen species; RPLC: reverse-phase liquid chromatography; UHPLCHRMS: ultra-high performance liquid chromatography- high-resolution mass spectrometry.

\section{Declarations}

\section{Acknowledgements}

The mass spectrometry analysis was supported by the Academia Sinica Metabolomics Core Facility at the Agricultural Biotechnology Research Center of Academia Sinica, supported by Academia Sinica Core Facility and Innovative Instrument Project (AS-CFII-111-218). The authors gratefully acknowledge the mass spectrometry analysis supported by the National Taiwan University Consortia of Key Technologies and National Taiwan University Instrumentation center, and ICP00401 and MS004000 equipment belonging to the Core Facility Center of National Cheng Kung University.

\section{Funding}

This work was supported by the Ministry of Science and Technology, Taiwan [grant number MOST1092113-M-006-015 and MOST110-2113-M-006-014]. 


\section{Availability of data and materials}

All data generated or analyzed during this study are included in this published article.

\section{Authors' contributions}

CWC: conducted hair metabolite extraction experiments, data analysis and manuscript writing. SWL: conducted hair metabolite extraction experiments and manuscript writing. PSS, RHW, and CHW: contributed to recruit the participants in National Cheng Kung University Hospital. YCC: contributed to manuscript writing. PCL: supervised the design and execution of the study and the writing of the manuscript. All authors read and approved the final manuscript.

\section{Ethics approval and consent to participate}

This study was approved by the Institutional Review Board of National Cheng Kung University Hospital (IRB approval no. B-ER-108-188).

\section{Consent for publication}

Not applicable

\section{Competing interests}

The authors have declared that no competing interest exists.

\section{Author details}

${ }^{1}$ Department of Environmental and Occupational Health, College of Medicine, National Cheng Kung University, Tainan, Taiwan. ${ }^{2}$ Department of Neurology, National Cheng Kung University Hospital, College of Medicine, National Cheng Kung University, Tainan, Taiwan. ${ }^{3}$ Institute of Gerontology, College of Medicine, National Cheng Kung University, Tainan, Taiwan. ${ }^{4}$ Department of Family Medicine, College of Medicine, National Cheng Kung University, Tainan, Taiwan. ${ }^{5}$ Department of Family Medicine, National Cheng Kung University Hospital, College of Medicine, National Cheng Kung University, Tainan, Taiwan. ${ }^{6}$ Department of Food Safety/Hygiene and Risk Management, College of Medicine, National Cheng Kung University, Tainan, Taiwan.

\section{References}


1. Porsteinsson AP, Isaacson RS, Knox S, Sabbagh MN, Rubino I. Diagnosis of Early Alzheimer's Disease: Clinical Practice in 2021. The Journal of Prevention of Alzheimer's Disease. 2021;8(3):37186.

2. Hane FT, Robinson M, Lee BY, Bai O, Leonenko Z, Albert MS. Recent Progress in Alzheimer's Disease Research, Part 3: Diagnosis and Treatment. J Alzheimers Dis. 2017;57(3):645-65.

3. Hajjar I, Liu C, Jones DP, Uppal K. Untargeted metabolomics reveal dysregulations in sugar, methionine, and tyrosine pathways in the prodromal state of AD. Alzheimers Dement (Amst). 2020;12(1):e12064.

4. Jack CR, Jr., Lowe VJ, Senjem ML, Weigand SD, Kemp BJ, Shiung MM, et al. 11C PiB and structural MRI provide complementary information in imaging of Alzheimer's disease and amnestic mild cognitive impairment. Brain. 2008;131(Pt 3):665-80.

5. Bayer AJ. The role of biomarkers and imaging in the clinical diagnosis of dementia. Age Ageing. 2018;47(5):641-3.

6. Wattmo C, Blennow K, Hansson O. Cerebrospinal Fluid Biomarker Levels as Markers for Nursing Home Placement and Survival Time in Alzheimer's Disease. Curr Alzheimer Res. 2021.

7. Alonso A, Marsal S, Julià A. Analytical methods in untargeted metabolomics: state of the art in 2015. Front Bioeng Biotechnol. 2015;3:23-.

8. Wishart DS, Feunang YD, Marcu A, Guo AC, Liang K, Vázquez-Fresno R, et al. HMDB 4.0: the human metabolome database for 2018. Nucleic Acids Res. 2018;46(D1):D608-d17.

9. Shao Y, Ouyang Y, Li T, Liu X, Xu X, Li S, et al. Alteration of Metabolic Profile and Potential Biomarkers in the Plasma of Alzheimer's Disease. Aging Dis. 2020;11(6):1459-70.

10. Chouraki V, Preis SR, Yang Q, Beiser A, Li S, Larson MG, et al. Association of amine biomarkers with incident dementia and Alzheimer's disease in the Framingham Study. Alzheimers Dement. 2017;13(12):1327-36.

11. Slupsky CM, Rankin KN, Wagner J, Fu H, Chang D, Weljie AM, et al. Investigations of the Effects of Gender, Diurnal Variation, and Age in Human Urinary Metabolomic Profiles. Analytical Chemistry. 2007;79(18):6995-7004.

12. Saude EJ, Adamko D, Rowe BH, Marrie T, Sykes BD. Variation of metabolites in normal human urine. Metabolomics. 2007;3(4):439-51.

13. Giskeødegård GF, Davies SK, Revell VL, Keun H, Skene DJ. Diurnal rhythms in the human urine metabolome during sleep and total sleep deprivation. Sci Rep. 2015;5:14843-.

14. Sulek K, Han TL, Villas-Boas SG, Wishart DS, Soh SE, Kwek K, et al. Hair metabolomics: identification of fetal compromise provides proof of concept for biomarker discovery. Theranostics. 2014;4(9):9539.

15. Delplancke TDJ, de Seymour JV, Tong C, Sulek K, Xia Y, Zhang H, et al. Analysis of sequential hair segments reflects changes in the metabolome across the trimesters of pregnancy. Scientific Reports. 2018;8(1):36. 
16. Yang J, Wei Y, Qi H, Yin N, Yang Y, Li Z, et al. Neonatal hair profiling reveals a metabolic phenotype of monochorionic twins with selective intrauterine growth restriction and abnormal umbilical artery flow. Mol Med. 2020;26(1):37.

17. Harkey MR. Anatomy and physiology of hair. Forensic science international. 1993;63(1-3):9-18.

18. Sauve B, Koren G, Walsh G, Tokmakejian S, Van Uum SH. Measurement of cortisol in human hair as a biomarker of systemic exposure. Clinical and investigative medicine Medecine clinique et experimentale. 2007;30(5):E183-91.

19. Vogliardi S, Tucci M, Stocchero G, Ferrara SD, Favretto D. Sample preparation methods for determination of drugs of abuse in hair samples: A review. Anal Chim Acta. 2015;857:1-27.

20. Henderson GL. Mechanisms of drug incorporation into hair. Forensic Science International. 1993;63(1):19-29.

21. Jang WJ, Choi JY, Park B, Seo JH, Seo YH, Lee S, et al. Hair Metabolomics in Animal Studies and Clinical Settings. Molecules. 2019;24(12).

22. Chang WC-W, Wang P-H, Chang C-W, Chen Y-C, Liao P-C. Extraction strategies for tackling complete hair metabolome using LC-HRMS-based analysis. Talanta. 2021;223:121708.

23. Cooper GA, Kronstrand R, Kintz P. Society of Hair Testing guidelines for drug testing in hair. Forensic Sci Int. 2012;218(1-3):20-4.

24. Pang Z, Chong J, Zhou G, de Lima Morais DA, Chang L, Barrette M, et al. MetaboAnalyst 5.0: narrowing the gap between raw spectra and functional insights. Nucleic Acids Res. 2021;49(W1):W388-w96.

25. Tsugawa H, Kind T, Nakabayashi R, Yukihira D, Tanaka W, Cajka T, et al. Hydrogen Rearrangement Rules: Computational MS/MS Fragmentation and Structure Elucidation Using MS-FINDER Software. Analytical Chemistry. 2016;88(16):7946-58.

26. Morris JC. Clinical dementia rating: a reliable and valid diagnostic and staging measure for dementia of the Alzheimer type. Int Psychogeriatr. 1997;9 Suppl 1:173-6; discussion 7-8.

27. Schober P, Boer C, Schwarte LA. Correlation Coefficients: Appropriate Use and Interpretation. Anesthesia \& Analgesia. 2018;126(5).

28. Barbosa J, Faria J, Carvalho F, Pedro M, Queirós O, Moreira R, et al. Hair as an alternative matrix in bioanalysis. Bioanalysis. 2013;5(8):895-914.

29. Psychogios N, Hau DD, Peng J, Guo AC, Mandal R, Bouatra S, et al. The human serum metabolome. PloS one. 2011;6(2):e16957-e.

30. Rappaport SM, Barupal DK, Wishart D, Vineis P, Scalbert A. The blood exposome and its role in discovering causes of disease. Environ Health Perspect. 2014;122(8):769-74.

31. Barupal DK, Fiehn O. Generating the Blood Exposome Database Using a Comprehensive Text Mining and Database Fusion Approach. Environ Health Perspect. 2019;127(9):97008.

32. Tricco AC, Ashoor HM, Soobiah C, Rios P, Veroniki AA, Hamid JS, et al. Comparative Effectiveness and Safety of Cognitive Enhancers for Treating Alzheimer's Disease: Systematic Review and Network 
Metaanalysis. Journal of the American Geriatrics Society. 2018;66(1):170-8.

33. Di Costanzo A, Paris D, Melck D, Angiolillo A, Corso G, Maniscalco M, et al. Blood biomarkers indicate that the preclinical stages of Alzheimer's disease present overlapping molecular features. Sci Rep. 2020;10(1):15612.

34. Nagata Y, Hirayama A, Ikeda S, Shirahata A, Shoji F, Maruyama M, et al. Comparative analysis of cerebrospinal fluid metabolites in Alzheimer's disease and idiopathic normal pressure hydrocephalus in a Japanese cohort. Biomark Res. 2018;6:5.

35. Cristofano A, Sapere N, La Marca G, Angiolillo A, Vitale M, Corbi G, et al. Serum Levels of AcylCarnitines along the Continuum from Normal to Alzheimer's Dementia. PLOS ONE. 2016;11(5):e0155694.

36. Lin C-N, Huang C-C, Huang K-L, Lin K-J, Yen T-C, Kuo H-C. A metabolomic approach to identifying biomarkers in blood of Alzheimer's disease. Annals of Clinical and Translational Neurology. 2019;6(3):537-45.

37. Li Z, Vance DE. Phosphatidylcholine and choline homeostasis. J Lipid Res. 2008;49(6):1187-94.

38. Walter A, Korth U, Hilgert M, Hartmann J, Weichel O, Hilgert M, et al. Glycerophosphocholine is elevated in cerebrospinal fluid of Alzheimer patients. Neurobiol Aging. 2004;25(10):1299-303.

39. Mapstone M, Cheema AK, Fiandaca MS, Zhong X, Mhyre TR, MacArthur LH, et al. Plasma phospholipids identify antecedent memory impairment in older adults. Nature Medicine. 2014;20(4):415-8.

40. Whiley L, Sen A, Heaton J, Proitsi P, García-Gómez D, Leung R, et al. Evidence of altered phosphatidylcholine metabolism in Alzheimer's disease. Neurobiol Aging. 2014;35(2):271-8.

41. Mulder C, Wahlund LO, Teerlink T, Blomberg M, Veerhuis R, van Kamp GJ, et al. Decreased lysophosphatidylcholine/phosphatidylcholine ratio in cerebrospinal fluid in Alzheimer's disease. J Neural Transm (Vienna). 2003;110(8):949-55.

42. Jia L, Yang J, Zhu M, Pang Y, Wang Q, Wei Q, et al. A metabolite panel that differentiates Alzheimer's disease from other dementia types. Alzheimers Dement. 2021.

43. Wang C, Cai Z, Wang W, Wei M, Kou D, Li T, et al. Piperine attenuates cognitive impairment in an experimental mouse model of sporadic Alzheimer's disease. J Nutr Biochem. 2019;70:147-55.

44. Laux-Biehlmann A, Mouheiche J, Vérièpe J, Goumon Y. Endogenous morphine and its metabolites in mammals: history, synthesis, localization and perspectives. Neuroscience. 2013;233:95-117.

45. Surh Y. Tetrahydropapaveroline, a dopamine-derived isoquinoline alkaloid, undergoes oxidation: implications for DNA damage and neuronal cell death. Eur J Clin Invest. 1999;29(7):650-1.

46. Lee JJ, Kim YM, Yin SY, Park HD, Kang MH, Hong JT, et al. Aggravation of L-DOPA-induced neurotoxicity by tetrahydropapaveroline in PC12 cells. Biochem Pharmacol. 2003;66(9):1787-95.

47. Ansari MA, Scheff SW. Oxidative stress in the progression of Alzheimer disease in the frontal cortex. J Neuropathol Exp Neurol. 2010;69(2):155-67. 
48. Nunomura A, Perry G, Aliev G, Hirai K, Takeda A, Balraj EK, et al. Oxidative damage is the earliest event in Alzheimer disease. J Neuropathol Exp Neurol. 2001;60(8):759-67.

49. Soh Y, Shin MH, Lee JS, Jang JH, Kim OH, Kang H, et al. Oxidative DNA damage and glioma cell death induced by tetrahydropapaveroline. Mutat Res. 2003;544(2-3):129-42.

50. Surh Y-J, Kim H-J. Neurotoxic effects of tetrahydroisoquinolines and underlying mechanisms. Exp Neurobiol. 2010;19(2):63-70.

51. Charron G, Doudnikoff E, Laux A, Berthet A, Porras G, Canron MH, et al. Endogenous morphine-like compound immunoreactivity increases in parkinsonism. Brain. 2011;134(Pt 8):2321-38.

52. Nowicki M, Tran S, Chatterjee D, Gerlai R. Inhibition of phosphorylated tyrosine hydroxylase attenuates ethanol-induced hyperactivity in adult zebrafish (Danio rerio). Pharmacol Biochem Behav. 2015;138:32-9.

53. Peana AT, Bassareo V, Acquas E. Not Just from Ethanol. Tetrahydroisoquinolinic (TIQ) Derivatives: from Neurotoxicity to Neuroprotection. Neurotoxicity Research. 2019;36(4):653-68.

54. Antkiewicz-Michaluk L, Lazarewicz JW, Patsenka A, Kajta M, Zieminska E, Salinska E, et al. The mechanism of 1,2,3,4-tetrahydroisoquinolines neuroprotection: the importance of free radicals scavenging properties and inhibition of glutamate-induced excitotoxicity. Journal of Neurochemistry. 2006;97(3):846-56.

55. Nappi AJ, Vass E, Collins MA. Contrasting effects of catecholic and O-methylated tetrahydroisoquinolines on hydroxyl radical production. Biochimica et Biophysica Acta (BBA) Protein Structure and Molecular Enzymology. 1999;1434(1):64-73.

56. Park SJ, Lee J, Lee S, Lim S, Noh J, Cho SY, et al. Exposure of ultrafine particulate matter causes glutathione redox imbalance in the hippocampus: A neurometabolic susceptibility to Alzheimer's pathology. Science of The Total Environment. 2020;718:137267.

\section{Tables}

\section{Table 1}

Demographics of Alzheimer's disease (AD) patients and control subjects. 


\section{Characteristic}

AD patients

Healthy Control

p-value

Sex

Female

Male

Age (years)

Mean \pm SD

$68.7 \pm 6.5$

$66.2 \pm 6.2$

0.17

\section{BMI $\left(\mathrm{kg} / \mathrm{m}^{2}\right)$}

Mean \pm SD

$24.2 \pm 4.0$

$23.9 \pm 3.2$

0.83

MoCA score

Mean \pm SD

$15.5 \pm 5.3$

$27.4 \pm 1.4$

$<0.0001$

CDR score

0.5

1

2

Cosmetic hair treatment

Perming
Never

Dyeing

Both

Smoking status

Never

21

3

Past

Current

Alcohol consumption

Never

23

0

1

15

0.93

3

6

2

2

Past

Current

Note: AD patients were subjected to cognitive examinations (MoCA and CDR) and diagnosed based on professional physicians from the Department of Neurology. Control subjects were subjected to MoCA examination. 
Student's t test was applied to calculate the statistical significance of age, BMI and MoCA score between AD and controls, and Chi-square test was used to calculate the statistical significance of cosmetic hair treatment, smoking status and alcohol consumption

\section{Table 2}

Statistically significant metabolites between 14 mild AD (CDR score 0.5 ) and 24 control using untargeted metabolomics analysis.

\begin{tabular}{lllll} 
PubChem CID & Metabolite name & Mild AD / Control (a) & P value $^{(\mathbf{b})}$ & AUC (c) \\
\hline 182440 & 6-O-methylnorlaudanosoline & 0.16 & 0.0056 & 0.86 \\
\hline 638024 & Piperine & 0.36 & 0.0026 & 0.82 \\
\hline 213144 & Butyrylcarnitine & 0.40 & 0.0098 & 0.78 \\
\hline 7045767 & Acetyl-L-carnitine & 0.41 & 0.0029 & 0.77 \\
\hline 107738 & Propionylcarnitine & 0.36 & 0.0094 & 0.75 \\
\hline 21777566 & Hydroxyprolyl-Leucine & 0.27 & 0.0046 & 0.75 \\
\hline 24779465 & LPC 18:1 & 0.41 & 0.0081 & 0.74 \\
\hline 460602 & PC (16:0/0:0) & 0.42 & 0.0020 & 0.73 \\
\hline 16226475 & O-valeroyl-L-carnitine & 0.45 & 0.0071 & 0.73 \\
\hline 1050 & Pyridoxal & 0.35 & 0.0230 & 0.73 \\
\hline
\end{tabular}

(a) Average of peak abundance of metabolites in mild AD patients (CDR score 0.5 ) divided by that in controls (b) All measurements were analyzed by Student t-test and $\mathrm{p}$-value $<0.05$ indicates a statistically significant difference (c) AUC of mild AD versus control.

\section{Figures}




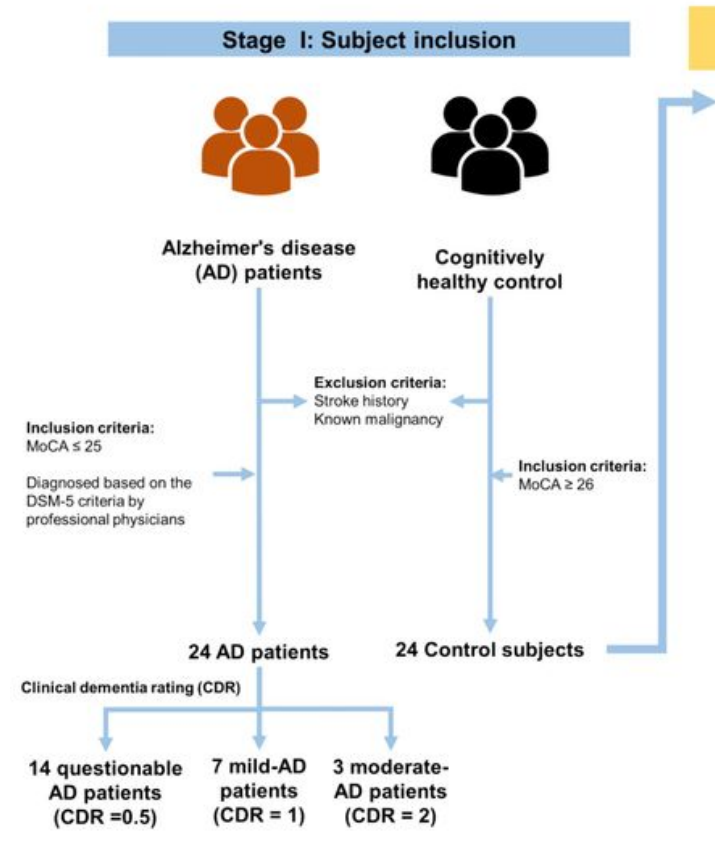

Stage II: Discovery and identification of biomarkers

Hair collection and extraction

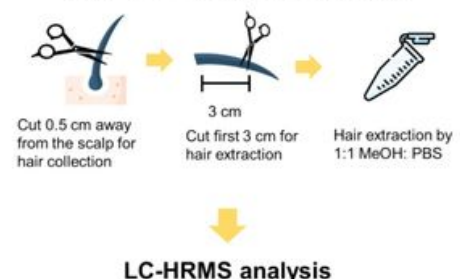

LC-HRMS analysis

Data processing

Volcano plot analysis Fold change $>2$ or $<0.5$ $p$ value $<0.01$ (Student's t test)

Chemical structure identification
Stage III: Applications of biomarkers

Patients with different severities of $A D$ and

cognitively healthy controls

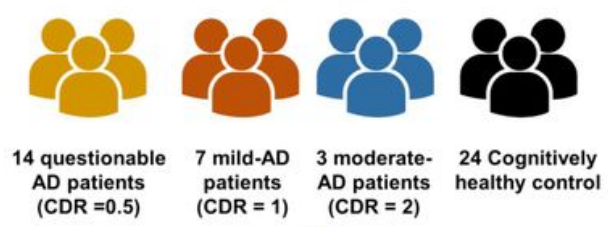

Levels of metabolites in different groups

ROC curve analysis

\section{Figure 1}

Study design of hair biomarkers of Alzheimer's disease (AD) discovery and interpretation using a highresolution mass spectrometry (HRMS) based untargeted metabolomics approach. A total of $24 \mathrm{AD}$ patients and 24 control subjects without stroke history and known malignancy were recruited in this study. All participants underwent MoCA test, and the control subjects included the MoCA score higher than 26. The AD patient underwent further CDR assessment to quantify the severity of the symptoms of dementia. The extraction of hair samples followed the developed method and subsequently analyzed in both positive- and negative- ion mode using RPLC-HRMS. Biomarker candidates were discovered by the criteria (fold change $\geq 2$ or $\leq 0.5, p \leq 0.01$ ) using volcano plot. 
A

$A D$ vs Control

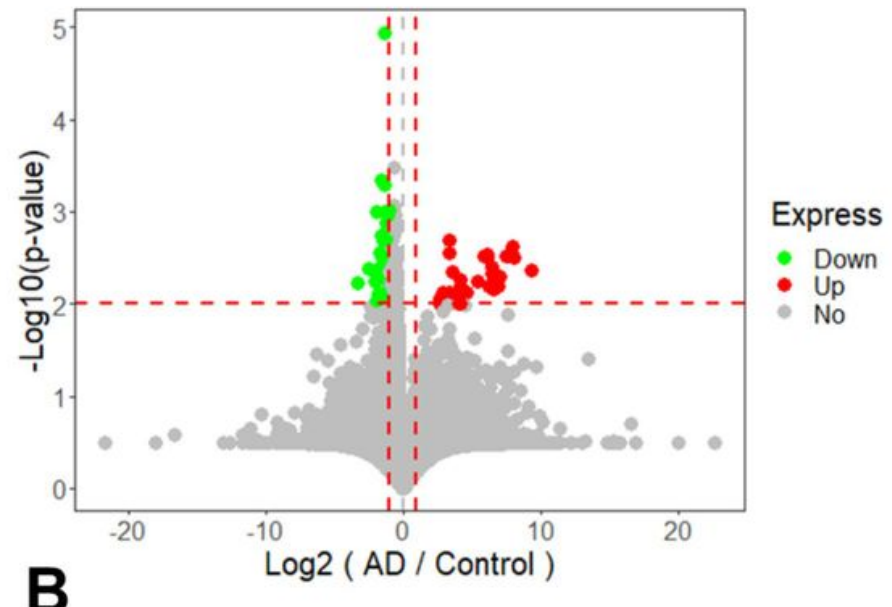

$A D$ vs Control

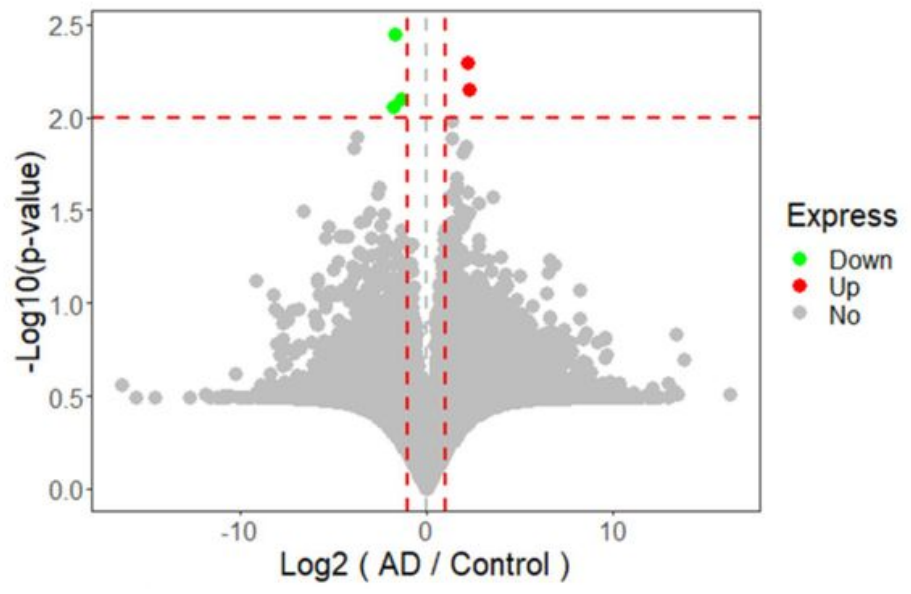

Figure 2

Statistically significant features ( $A D /$ Control $\geq 2$ or $\leq 0.5, p \leq 0.01)$ in the hair between Alzheimer's disease and controls. (A)The 67 of 12,353 detected signals were discriminatory features (fold change $\geq 2$ or $\leq 0.5, p \leq 0.01$ ) in positive ion modes. Of these, abundances of 33 signals were increased and 38 signals were decreased in $A D$ group relative to control in positive ion mode. (B) The 14 of 8,821 signals were significance using the criteria (fold change $\geq 2$ or $\leq 0.5, p \leq 0.01$ ) in negative ion mode. Of those, 10 signals were overexpressed, and 4 signals were under expressed in $A D$ patients relative to control in negative ion mode analysis. 


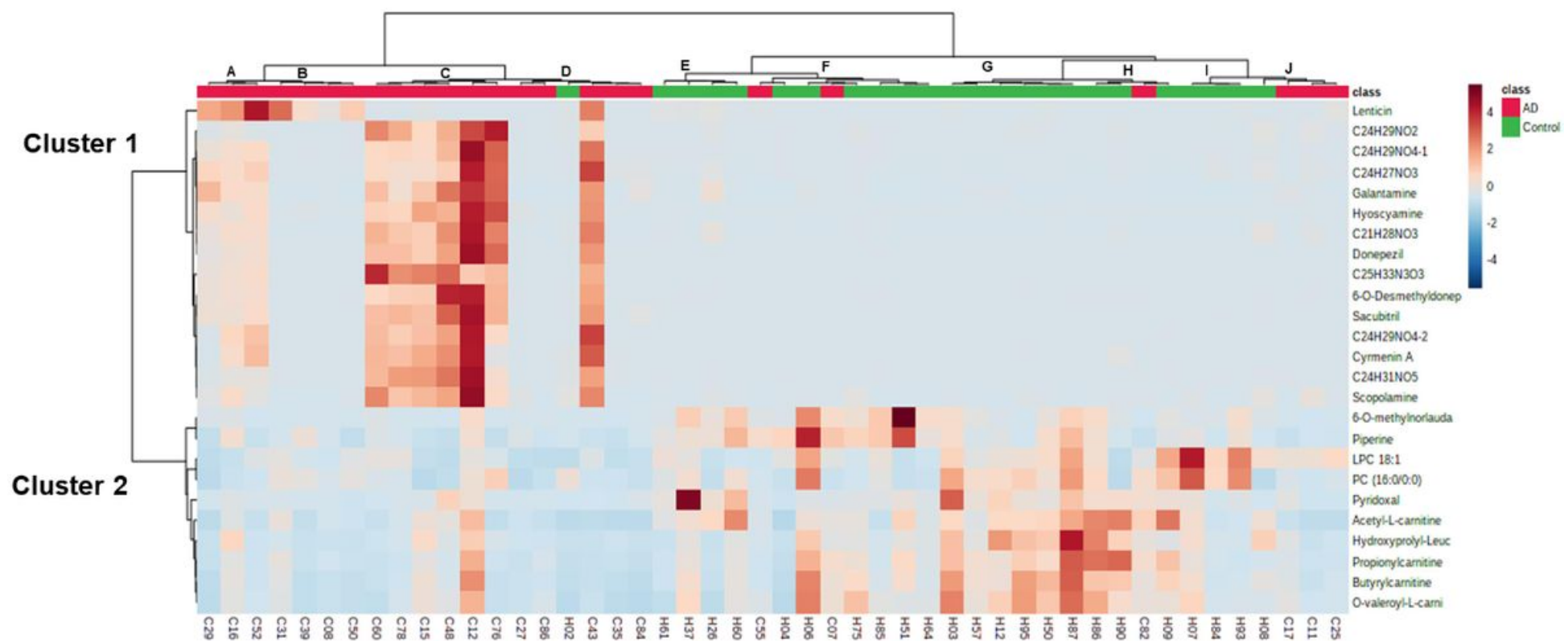

Figure 3

Two-way hierarchical clustering analysis (HCA) of $\mathbf{2 5}$ annotated metabolites in $\mathbf{4 8}$ hair samples. The 48 hair samples could be grouped into 10 clusters (Clusters A J) with similar peak feature profiles, and 25 annotated metabolites could be primarily grouped into 2 clusters (Clusters 1 and 2). Extensive contents of the discriminatory features are represented by the red pixels in the heatmap, whereas bright blue and intense blue represent average and low content, respectively. 
A

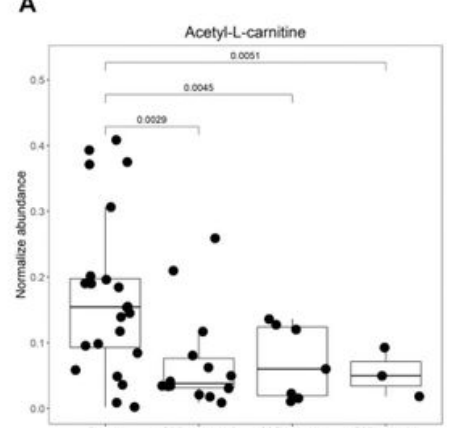

D

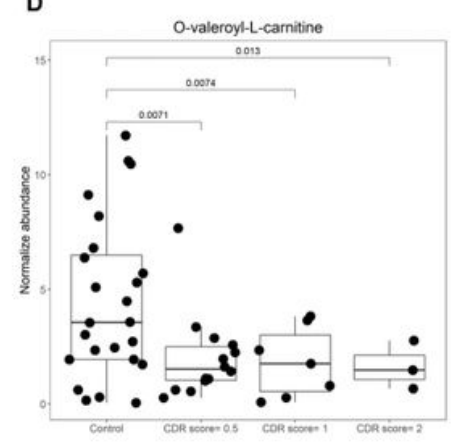

G

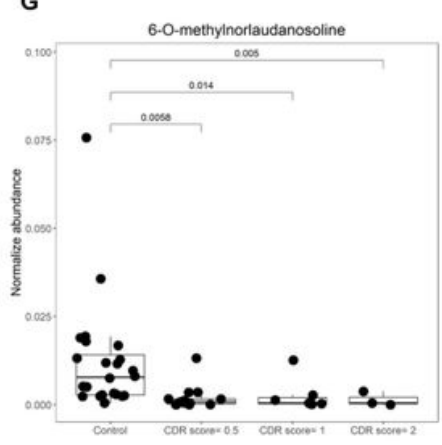

B

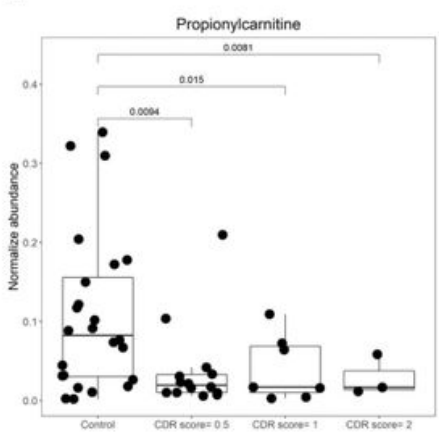

E

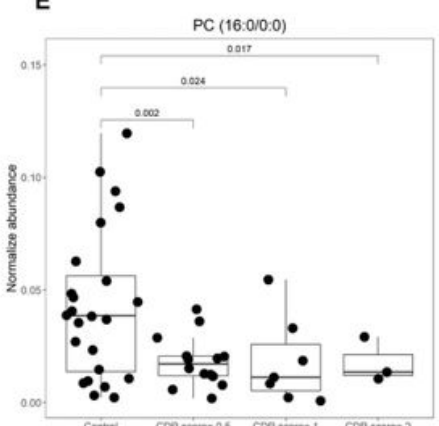

H

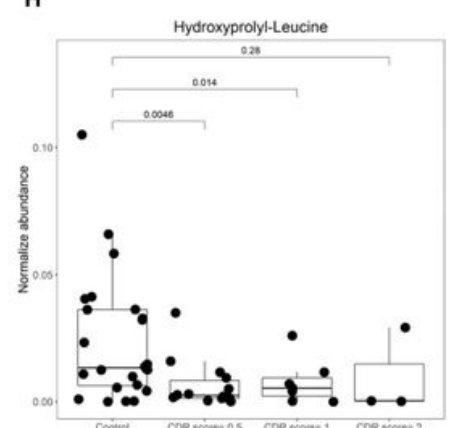

C

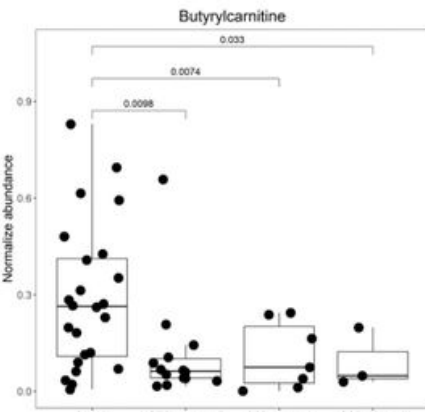

F

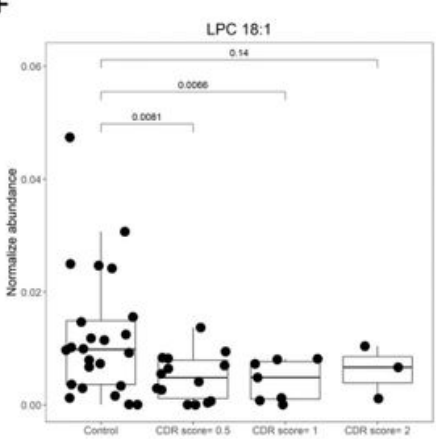

I

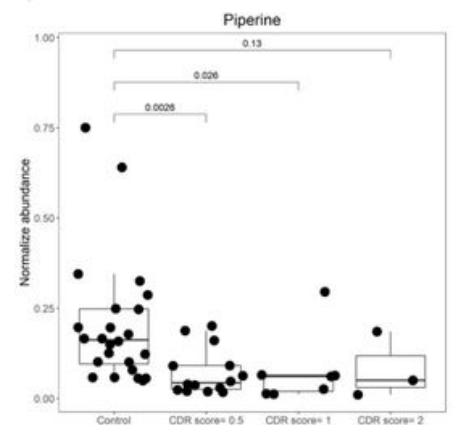

Figure 4

Scatter and box plots of candidate biomarker metabolites for early detection of $A D$ dementia in the derived panel. The metabolites, (A) acetyl-L-carnitine, (B) propionylcarnitine, (C) butyrylcarnitine, (D) Ovaleroyl-L-carnitine, (E) PC (16:0/0:0), (F) LPC 18:1, (G) 6-0-methylnorlaudanosoline, (H) hydroxyprolylleucine, and (I) piperine, represent the normalized metabolites intensity. 


\section{Questionable AD vs \\ Control}

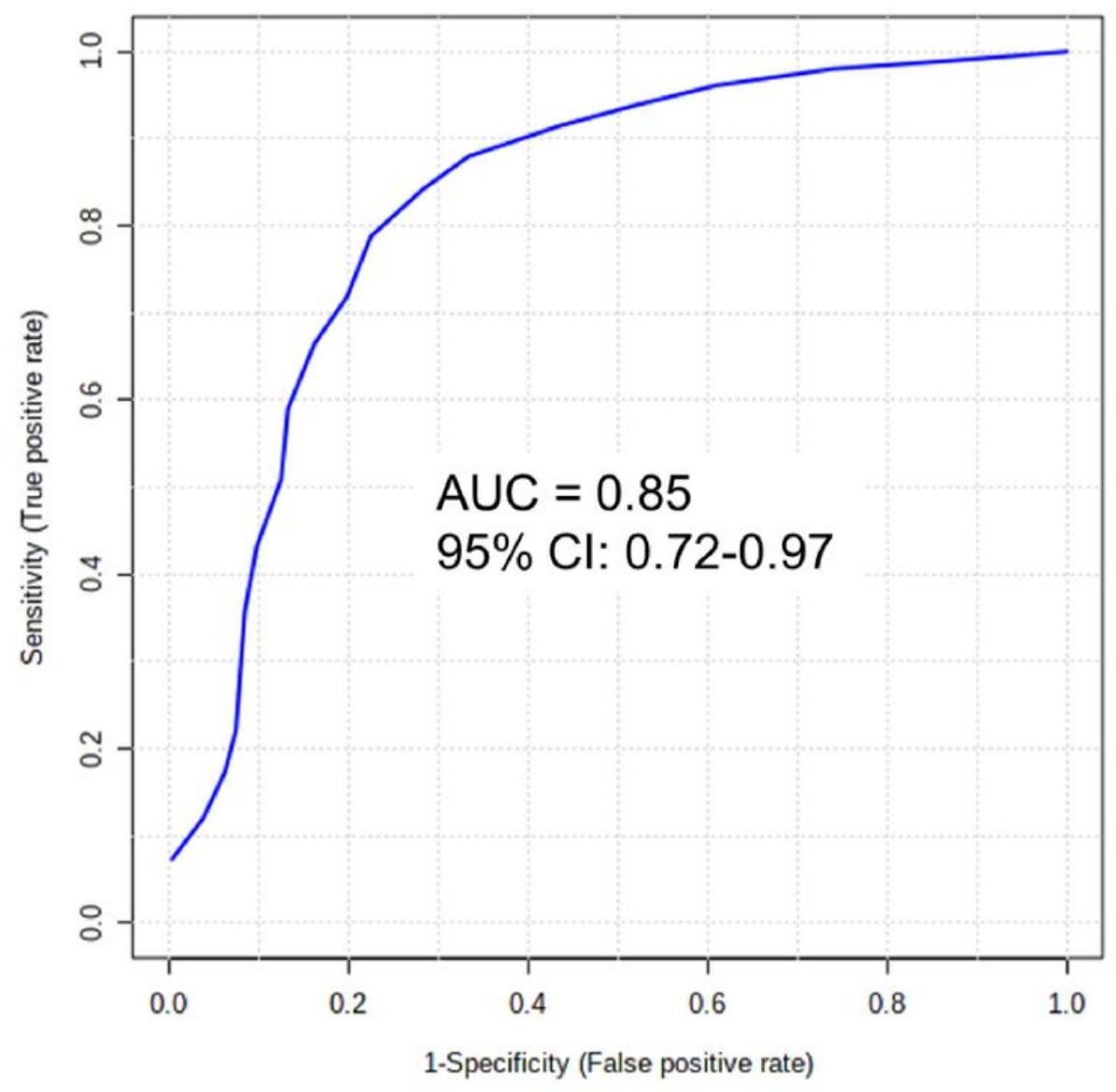

Figure 5

Receiver operator characteristic (ROC) curve analysis of statistically significant metabolites in the hair between patients with questionable AD (CDR score of 0.5 ) and controls. ROC curve analysis was performed to compare the predictive power of mild AD and control with combination of 9 metabolites using random forest.

\section{Supplementary Files}

This is a list of supplementary files associated with this preprint. Click to download.

- Additionalfile.docx 